\title{
la haine peut-elle être un sentiment républicain ? À propos du serment civique de l'an $\mathrm{V}$
}

Can hatred be a Republican sentiment? The civic oath of the year $V$

\section{Marc Deleplace}

\section{(2) OpenEdition \\ 1 Journals}

\section{Édition électronique}

URL : https://journals.openedition.org/ahrf/11527

DOI : 10.4000/ahrf.11527

ISSN : 1952-403X

Éditeur :

Armand Colin, Société des études robespierristes

\section{Édition imprimée}

Date de publication : 1 octobre 2009

Pagination : 47-70

ISBN : 978-2-200-92560-4

ISSN : 0003-4436

\section{Référence électronique}

Marc Deleplace, « la haine peut-elle être un sentiment républicain ? À propos du serment civique de l'an V », Annales historiques de la Révolution française [En ligne], 358 | octobre-décembre 2009, mis en ligne le 01 octobre 2012, consulté le 23 avril 2022. URL : http://journals.openedition.org/ahrf/11527 ; DOl : https://doi.org/10.4000/ahrf.11527 


\title{
LA HAINE PEUT-ELLE ÊTRE UN SENTIMENT RÉPUBLICAIN? A PROPOS DU SERMENT CIVIQUE DE L'ANV
}

\begin{abstract}
Poser la question de la haine comme sentiment républicain peut sembler paradoxal. C'est pourtant bien ainsi qu'elle surgit au cœur de l'archive par le serment de haine prêté doublement, contre la royauté et contre l'anarchie, par les autorités républicaines entre l'an $\mathrm{V}$ et l'an VII. Notre réflexion sur ce serment emprunte ainsi un double chemin. D'une part, elle invite à considérer comment la question de l'ordre politique peut se réfléchir en termes de sentiment. De l'autre, elle soulève la question des procédures par lesquelles les républicains du Directoire ont pu penser fonder l'ordre social sur un sentiment de haine.
\end{abstract}

Mots-clés : haine, Directoire, serment

La question ainsi posée peut sembler doublement provocatrice, si on la rapproche du projet d'éducation républicaine de la III ${ }^{\mathrm{e}}$ République, dans la mesure où ce projet repose d'une part sur le développement d'un individualisme éclairé qui assure à chaque citoyen la maîtrise de ses affects dans une perspective de rationalité politique ${ }^{1}$ susceptible d'endiguer le déchaînement des passions dans l'espace public ${ }^{2}$, et qu'il attribue d'autre part à l'enseignement de l'histoire la capacité à éteindre les haines

(1) Yves DéloYe, École et citoyenneté : l'individualisme républicain de Jules Ferry à Vichy, controverses, Paris, Presses de la FNSP, 1994.

(2) Nous utilisons ce terme en référence à la notion d'espace public de réciprocité. Jürgen Habermas, L'Espace public. Archéologie de la publicité comme dimension de la société bourgeoise, 1962, trad. fr., Paris, Payot, 1978. 
héritées de la difficile gestation de la République. Telle est la position tenue par « l'instituteur de la République $»^{3}$, Ernest Lavisse lorsqu'il met en garde, contre une lecture trop courte de l'histoire de France, plus propre à éterniser le ressentiment politique qu'à l'apaiser, marquant par là même sa défiance à l'égard d'un enseignement sans précaution du moment révolutionnaire pourtant érigé par ailleurs en événement fondateur de la République. «L'homme du peuple en France, le paysan surtout, est l'homme le plus prosaïque du monde. [...] Montrons lui [...] qu'il y a des légitimités successives au cours de la vie d'un peuple et qu'on peut aimer toute la France sans manquer à ses obligations envers la République $»^{4}$. C'est que la Révolution, si elle fonde la République, s'accompagne d'un cortège de violence que les historiens de cette République apaisée, tentée par Cérès autant que par Marianne ${ }^{5}$, cherchent à conjurer du mieux qu'ils peuvent dans le récit qu'ils en établissent alors ${ }^{6}$.

Évoquer la haine comme sentiment républicain peut enfin passer pour un paradoxe discutable dans une République qui adopte définitivement comme devise un triptyque qui institue la fraternité en complément indissociable de la liberté et de l'égalité, une fraternité étendue dans son principe au-delà des bornes du corps civique national ${ }^{7}$. Comment dès lors oserait-on soutenir que la haine puisse être envisagée comme fondement de l'ordre politique républicain?

Et pourtant... Le 2 pluviôse an V (21 janvier 1797), « anniversaire de la juste punition du dernier tyran », le citoyen Barry, au nom de l'administration de Toulon, s'apprête à prononcer le serment civique que les Législateurs ont décidé d'imposer à l'ensemble des autorités constituées de la République. Avant la prestation, il explique le sens de ce serment

(3) Expression reprise de Pierre Nora (dir.), Les Lieux de mémoire, t. 1 : La République, Paris, Gallimard, 1984.

(4) Ernest LavisSE, «L'enseignement historique en Sorbonne et l'Éducation nationale », 1881, repris dans Ernest Lavisse, Questions d'enseignement national, Paris, A. Colin, 1885. Cité dans Christian Delacroix, François Dosse, Patrick Garcia, Les Courants historiques en France $19^{e}-20^{e}$ siècle, Paris, A. Colin, « collection U », 1999.

(5) Maurice Agulhon, Marianne au combat 1789-1880, Paris, Flammarion, 1979; Les Métamorphoses de Marianne. L'imagerie et la symbolique républicaines de 1914 à nos jours, Paris, Flammarion, 2001.

(6) Marc Deleplace, «L'Émeute révolutionnaire au travers de manuels de la III République », Mots. Les langages du politique, $\mathrm{n}^{\circ} 69$, juillet 2002, p. 47-64.

(7) C'est le sens de la déclaration de politique étrangère de Lamartine au nom du gouvernement républicain en mars 1848. Maurice Agulhon, 1848 ou l'apprentissage de la République, Nouvelle histoire de la France contemporaine, t. 8, Paris, Le Seuil, «Points histoire », 2002, $1^{\text {re }}$ éd. 1973. 
ainsi rédigé : « Je jure haine à la royauté, je jure haine à l'anarchie, je jure attachement et fidélité à la République et à la constitution de l'an III ». D'emblée, il l'inscrit dans l'horizon d'une théorie des passions qui instaure le couple amour/haine comme paradigme fondamental. Il part du premier terme pour assurer que «l'amour de la liberté est un sentiment aussi naturel à l'homme que l'amour de l'existence ». Traduit en termes politiques, cet amour se comprend comme attachement à la république, forme naturelle ${ }^{8}$ de l'association politique. Aussi ce serment est-il « un devoir spécial pour les fonctionnaires publics, car c'est à eux, honorés du choix et de la confiance du peuple, à donner l'exemple des sentiments républicains. Peut-on en effet aimer la liberté sans haïr la royauté son ennemie implacable ? $»^{9}$ L'examen des devoirs publics afférant à la position de fonctionnaire public peut donc s'exposer dans le registre affectif des sentiments, dont la signification politique, relevant donc de la structuration d'un espace public, ne fait aucun doute puisqu'ils sont expressément qualifiés de « républicains ». Ainsi encore, parmi ces sentiments, celui de haine peut-il être légitimé dans une alternative sans ambiguïté qui associe le couple passionnel amour/haine au couple politique liberté/royauté, une royauté derrière laquelle le principe plus sûrement antinomique de la liberté est bien celui de la tyrannie.

Au-delà du paradoxe induit par notre titre, la possibilité pour le républicain de l'an $\mathrm{V}$ de fonder le lien social sur un sentiment de haine semble donc assumée sans restriction. La transformation de notre question initiale, la haine peut-elle être un sentiment républicain, en affirmation attestée dans l'archive n'est pas, contrairement aux apparences, une pure provocation. Elle mérite cependant quelques explications.

Oui, la haine peut être un sentiment républicain, puisque les autorités de la République décident de fonder l'ordre républicain sur un serment de haine ; oui, la haine peut être un sentiment républicain, puisque le citoyen Barry s'exprime en ces termes lors de la prestation de ce serment. Pourtant, le serment ne cesse de faire débat depuis son institution en nivôse an $\mathrm{V}$ jusqu'à sa réécriture en thermidor de l'an VII, réécriture

(8) Nous entendons « naturelle » dans son sens jusnaturaliste, car tel est l'horizon du propos de Barry, alors même que la constitution de l'an III qu'il s'agit ici de garantir, vient d'abandonner explicitement la référence au droit naturel dans ses principes. Seuls les droits de l'homme en société sont proclamés dans la Déclaration de l'an III. Voir Florence GaUTHIER, Triomphe et mort du droit naturel en Révolution (1789-1795-1802), Paris, PUF, « Pratiques théoriques », 1992, p. 247-260.

(9) Discours prononcé à la fête du 2 pluviôse an $5^{\mathrm{e}}$ de la république, avant la prestation $d u$ serment de haine à la royauté et à l'anarchie, et de fidélité à la constitution de l'an $3^{\mathrm{e}}$. Par le citoyen Barry, président de l'administration municipale du canton de Toulon, BN 8-LB42-1233. 
qui en fera précisément disparaître la haine. Il n'est sans doute pas vain de se demander comment les républicains directoriaux ont pu envisager de fonder la République sur un serment de haine, c'est-à-dire à la fois ce qui les a autorisés à concevoir un tel serment et les dispositions pratiques de sa mise en œuvre. Le serment, ainsi entendu comme pièce maîtresse de la légitimation républicaine en l'an V, soulève en fait plusieurs questions, qui ne sont pas seulement objet de débat pour l'historien du fait politique mais font débat tout au long du Directoire. Il suppose d'une part d'élucider rapidement les présupposés lexicaux sur lesquels reposent les débats qui se succèdent depuis l'adoption du serment jusqu'à sa reformulation, de l'autre de faire émerger les procès argumentaires qui articulent ces mêmes débats ${ }^{10}$.

\section{Haine et politique, vocabulaire des passions et espace public : questions de méthode}

Introduire la question des passions dans l'analyse historique de l'ordre politique en suggérant que c'est là chose inhabituelle et problématique peut apparaître bien naïf, tant la littérature historique, et pas nécessairement ancienne, nous a accoutumé au spectacle toujours renouvelé du jeu des passions traduites en déchaînements de violence, désarmant l'observateur, décourageant parfois l'analyse historique... Mais ne seraient-ce pas que des manifestations de réalités plus profondes? De ces réalités socio-économiques ou anthropologiques que l'histoire sociale du demisiècle écoulé au moins, dans toute sa richesse et sa diversité, nous a appris à préférer aux « agitations de surface ». Agitations d'abord rendues à leur insignifiance (en termes d'analyse historique entendons-nous bien) avant de trouver une consistance nouvelle comme objet historique ${ }^{11}$.

Cependant, le déchaînement violent des passions individuelles qui troublent ou bouleversent l'ordre social, le précipitant dans l'anarchie,

(10) On trouvera les premiers éléments de cette réflexion dans Marc Deleplace, « Peut-on fonder la république sur la haine ? Une interrogation sur la république directoriale », dans Frédéric Chauvaud et Ludovic Gaussot, Histoire et actualité de la haine, Rennes, Presses universitaires de Rennes, 2008 .

(11) Voir par exemple, dans des perspectives différentes : Jean Nicolas, La Rébellion française. Mouvements populaires et conscience sociale 1661-1789, Paris, Éditions du Seuil, 2002 ; Roger Dupuy, La politique du peuple. Racines, permanences et ambiguïtés du populisme XVIII XXe siècle, Paris, Albin Michel, 2002 ; Jean-Clément Martin, Violence et révolution. Essai sur la naissance d'un mythe national, Paris, Seuil, «L'univers historique », 2006. 
n'est pas le seul biais par lequel les passions font irruption dans l'analyse historique. Mais là encore, l'histoire sociale n'a guère retenu des analyses qui plaçaient telle passion comme ressort de la compréhension de tel régime politique, au contraire de la philosophie politique. Des catégories comme celles de vertu ou d'honneur chez Montesquieu ou encore l'envie dont Tocqueville fait le moteur de la société démocratique ${ }^{12}$, n'offrent $a$ priori que peu de prise à la catégorisation historiographique. Or, dans le mouvement qui accompagne ce qu'il serait peut-être approximatif de qualifier de retour du paradigme de l'individu, la question du sensible retrouve une place nouvelle dans l'analyse historique ${ }^{13}$ et Sophie Wahnich n'a pas hésité à recourir à la catégorie «d'émotion » pour reprendre l'analyse de la Terreur révolutionnaire ${ }^{14}$. C'est dans une perspective voisine que nous abordons ici la question de la haine dans la période directoriale. Avec deux nuances cependant. D'une part, plutôt qu'introduire une catégorie métadiscursive telle «qu'émotion» nous préférons, pour contextualiser notre analyse, reprendre la catégorie contemporaine de « sentiment $\rangle^{15}$. D'autre part, la haine intervient en premier lieu comme objet ou comme catégorie descriptive. C'est donc par l'attestation dans l'archive que nous nous autorisons in fine le recours à ces catégories comme catégories interprétatives, avançant quelques éléments pour contribuer à une réflexion sur la constitution de la haine comme objet et catégorie historique. Enfin, ce n'est pas tant les manifestations de la haine que nous recherchons, ce n'est donc pas la violence révolutionnaire ou contre-révolutionnaire qui nous retient, mais les contextes d'expression en termes de haine, au plus près des usages du mot, nous interdisant toute qualification a posteriori et non attestée dans l'archive, position méthodologique qui découle de ce qui précède ${ }^{16}$.

Cela suppose une brève élucidation du contexte linguistique dans lequel s'inscrivent les débats que nous allons suivre. Disons d'emblée que

(12) Olivier Le Cour Grandmaison, Haine(s). Philosophie et politique, Paris, PUF, « Politique d'aujourd'hui », 2002, p. 273-279.

(13) Jacques Rancière, Le Partage du sensible, Paris, La Fabrique, 2000.

(14) Sophie Wahnich, «De l'économie émotive de la Terreur », Annales, Histoire, Sciences sociales, vol. $57, \mathrm{n}^{\circ} 4,2002$.

(15) Sophie Wahnich s'explique très précisément sur le choix contraire, note 14 de l'article cité.

(16) Une position méthodologique fondée dans la tradition française de l'analyse du discours en histoire et dont nous nous sommes déjà expliqué : Marc DelePlace, « De l'histoire des idées à l'histoire sociale : un exemple de parcours méthodologique en analyse de discours ", Revue d'histoire moderne et contemporaine, 43-2, avril-juin 1996. 
de ce point de vue, l'exposé des motifs du citoyen Barry présente quelques difficultés. Sa péroraison repose d'une part sur une alternative qui se comprend aisément dans le registre des passions : « Peut-on en effet aimer la liberté sans haïr la royauté son ennemie implacable $\rangle^{17}$; mais dont la traduction en termes de sentiments (républicains) n'est pas aussi évidente qu'il y paraît.

Le couple amour/haine est en effet au cœur des théories des passions du XVII ${ }^{e}$ et du XVIII ${ }^{e}$ siècle, que ces théories soient d'inspiration chrétienne, comme chez l'oratorien Senault ${ }^{18}$, ou laïcisées voire athéistes chez d'Holbach $^{19}$. Et la relation qui unit l'une à l'autre ces deux passions, considérées comme le principe de toutes les autres, ne laisse pas d'être ambivalente, oscillant entre l'affirmation de deux principes contraires et la reconnaissance de leur identité de nature, positions qui ne sont pas exclusives l'une de l'autre ${ }^{20}$. Ce n'est pas ici le lieu d'exposer plus au long les réflexions sur cette question. $\mathrm{Du}$ moins pouvons-nous souligner deux constantes qui posent problème relativement à notre propos : que les passions relèvent de l'intériorité de l'âme lors même que leurs manifestations retentissent dans l'espace public; que dans les classifications qui distinguent les passions bonnes et mauvaises, celles qui tirent vers le bien de celles qui poussent au mal, la haine est constamment rangée dans les secondes.

Le premier point ramène à la question, récurrente sous la Révolution, de l'articulation entre privé et public ${ }^{21}$. Sémantiquement, il semble bien que c'est l'ambiguïté entre les termes de «passion » et de « sentiment » qui permette le glissement de la passion privée au principe public,

(17) Discours prononcé à la fête du 2 pluviôse an $5^{\mathrm{e}}$ de la république, avant la prestation $d u$ serment de haine à la royauté et à l'anarchie, et de fidélité à la constitution de l'an 3e. Par le citoyen Barry, président de l'administration municipale du canton de Toulon, BN 8-LB42-1233.

(18) Jean-François Senault, De l'Usage des passions, Paris, C. Journel, 1660, $1^{\text {re éd. }} 1641$.

(19) Paul-Henri D'Holbach, La Morale universelle ou les Devoirs de l'homme fondés sur sa nature, Amsterdam, M.-M. Rey, 1776.

(20) «La haine n'est pas plus ingénieuse à nuire que l'amitié ne l'est à servir : on l'a dit ; $\&$ c'est peut-être une prudence de la nature. O amour, ô haine, elle a voulu que vous fussiez redoutables, parce que son but le plus grand \& le plus universel est la production des êtres $\&$ leur conservation. Si on examine les passions de l'homme, on trouvera leur énergie proportionnée à l'intérêt de la nature ». Encyclopédie ou Dictionnaire raisonné des sciences, des arts et des métiers (page $8: 26$ ) Diderot. Cité d'après la base de données ARTFL.

(21) Articulation qui prend en l'an III une dimension spécifique dès lors que la reconnaissance de la justesse de l'action publique prend comme origine l'examen des qualités privées, comme le montre Sergio Luzatto lorsque se pose la délicate question des responsabilités collectives et individuelles dans la Terreur, qui conditionne celles de l'immunité parlementaire et de l'amnistie. L'automne de la Révolution. Luttes et cultures politiques dans la France thermidorienne, Paris, Honoré Champion, 2001. Question que nous avons partiellement abordée dans L'Anarchie de Mably à Proudhon 1750-1850. Histoire d'une appropriation polémique, Lyon, ENS-éditions, 2001. 
la confusion entre l'intériorité du sentiment et l'adhésion publique comme manifestation d'extériorisation. Dans l'Encyclopédie, les passions sont identifiées aux « penchants, inclinations, désirs et aversions », tandis que le « sentiment » est placé comme synonyme grammatical de « pensée » et " d'opinion », dont il est dit qu'ils « sont tous les trois d'usage lorsqu'il ne s'agit que de la simple énonciation de ses idées $»^{22}$. Et le chevalier de Jaucourt poursuit en reconnaissant que «les sentiments sont un peu soumis à l'influence du cœur; il n'est pas rare de les voir conformes à ceux des personnes qu'on aime ». Or de son côté, Diderot définit la haine comme un «sentiment de tristesse \& de peine qu'un objet absent ou présent excite au fond de notre cœur $»^{23}$. De cette ambiguïté de la haine comme passion et/ou sentiment réside peut-être l'un des passages possibles vers la haine positive dans l'ordre politique. Si tous deux relèvent de l'intériorité, mais si la passion se manifeste lorsque le sentiment s'énonce, alors seul le sentiment permet d'inscrire la haine dans l'ordre rationnel, et cette rationalité est seule susceptible de construire l'espace public. Affirmer la haine comme sentiment permet ainsi de transmuter ce qui ne peut que relever des mouvements intérieurs de l'âme en principe organisateur de l'ordre politique. L'un et l'autre peuvent en venir à se confondre dans le renouvellement d'une citoyenneté de l'adhésion qui ne semble donc pas avoir été totalement oubliée alors que s'affirme la prééminence du gouvernement représentatif et la médiation de la souveraineté du peuple par les élections. «Les véritables républicains saisiront toujours avec transport l'occasion des époques les plus mémorables de la révolution pour manifester leurs sentiments, pour faire connaître leurs opinions et leurs principes [...] Eh ! quel est le Français, quel est le républicain, quel est l'ami de son pays, le partisan de la liberté, l'adorateur de la vertu qui ne le fasse dans son cœur à chaque instant de sa vie, le serment de haine à la royauté quand il parcourt cette longue carrière de crimes qui signalèrent le règne des rois $»^{24}$. Cette adéquation supposée entre sentiment intérieur et expression publique de ce sentiment est la condition nécessaire de réalisation du serment, de l'assurance de sa validité dès lors que l'on reconnaît à la loi le droit d'imposer le principe de l'adhésion sans pour autant qu'elle ne puisse en vérifier la sincérité, alors que de cette

(22) JAUCOURT, op. cit., « Pensée, Sentiment, Opinion », page $12: 309$.

(23) Diderot, op. cit., « Haine », page $8: 26$.

(24) Discours prononcé par le citoyen Joubert, président de l'administration centrale du département de la Seine, séance du 2 pluviôse an 6 de la République. À l'occasion de la prestation du serment de haine à la royauté et à l'anarchie. BN 8-LB42-1757. 
sincérité découle l'efficience du serment et donc sa justification. «Ce n'est pas non plus un sentiment qu'elle commande, c'est l'expression d'un sentiment qu'elle suppose dans nos âmes et qu'elle a le droit d'exiger de tous ceux qu'elle emploie au maintien de la liberté, et qui sont indignes des fonctions républicaines s'ils ne savent pas haïr les oppresseurs de l'humanité $»^{25}$.

Reste à examiner l'autre point qui justifie le serment : la possibilité d'une haine positive dans l'ordre politique républicain.

\section{Gestation du serment civique de haine à la royauté et à l'anarchie (an IV-an V)}

Le serment civique, prêté par le corps législatif et par les différentes autorités constituées de la République est institué véritablement lors de la commémoration de l'exécution du roi, le 2 pluviôse de l'an V (21 janvier 1797). Les présidents du Conseil des Cinq-Cents et du Conseil des Anciens le prononcent tous deux au nom des représentants du peuple : «Je jure haine à la royauté, haine à l'anarchie ; je jure attachement et fidélité à la République et à la constitution de l'an III ». La formule initiale ne prévoyait, dans la logique de l'événement, que le serment de haine à la royauté. Celui de haine à l'anarchie fut ajouté sur proposition de JeanFrançois Philippes-Deleville, le 22 nivôse an V, dans le climat de réaction anti-jacobine qui devait aboutir au succès royaliste de germinal, pour qu'il n'y eût point de confusion avec « les partisans de Marat et de Robespierre et leurs successeurs $»^{26}$.

Ce serment marque de la sorte un jalon dans la liquidation de l'expérience du Gouvernement révolutionnaire et réitère le refus de la Convention thermidorienne, attisé par les journées de prairial an III

(25) Hymne à l'Être suprême. Discours sur le serment de haine à la royauté et à l'anarchie et imprécation contre les parjures. Pour la fête du 2 pluviôse VII de la République Française, anniversaire du 21 janvier 1793 par le citoyen Mulot, ancien député du département de Paris à la première législature, professeur de belles lettres, membre du Lycée des Arts et de la société libre des sciences, lettres et arts de Paris [Mayence]. BN 8-LB42-2058.

(26) Motion d'ordre de Jean-François Philippes-Deleville, 22 nivôse an V, BN Le43 678. Georges LeFEBVRE, presque seul parmi les auteurs des grandes synthèses sur la Révolution, accorde un peu d'attention à ce serment. La Révolution française, $7^{e}$ édition revue et augmentée par Albert Soboul,

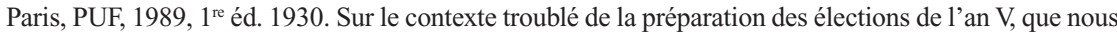
ne développons pas ici et dans lequel ce serment est institué, voir, outre l'article de Jean-René SURATTEAU, «Les élections de l'an V aux conseils du Directoire », Annales Historiques de la Révolution française, 1958, p. 21-63, Serge ABERDAM et alii, Voter, élire pendant la Révolution française 17891799, Paris, éditions du CTHS, 1999, et Malcolm CrooK, Elections in the French Revolution. An apprenticeship in democracy 1789-1799, Cambridge, Cambridge University Press, 1996. 
(mai 1795) et symbolisé par la constitution elle-même, de maintenir la constitution de 1793, au nom du « danger anarchique » qu'elle contenait, selon les propres termes de Boissy-d'Anglas en messidor de l'an III $^{27}$. Aussi n'est-il pas inutile, pour en saisir la portée et en comprendre l'adoption par le Corps Législatif, de revenir sur les circonstances dans lesquelles il intervient. Celles-ci touchent à la fois à ce que peut recouvrir l'expression de la haine en l'an V et à la recherche incessante d'un équilibre politique qui écarte aussi bien le spectre de la «tyrannie royale » que celui de la «tyrannie à bonnet rouge $»^{28}$. Une attitude qui soutient d'abord la politique des thermidoriens puis celle du Directoire, et qui trouve une acuité renforcée entre la conjuration des Égaux (21 floréal an IV-10 mai 1796) et le coup d'État du 18 fructidor an V (4 septembre 1797).

Si nous suivons les modalités par lesquelles le sentiment de haine a pu être valorisé en l'an $\mathrm{V}$ au point de s'imposer comme la pierre de touche de l'attachement républicain, nous ne pouvons faire l'économie d'usages négatifs plus habituels de l'expression de haine. Le discours politique thermidorien est ainsi riche de mentions de la haine vouée à la Révolution par ses adversaires. Ou, pour être plus exact, des haines de toute nature auxquelles la République est en butte. Haines attisées tout d'abord par les contre-révolutionnaires, dans une République en guerre avec les rois coalisés d'Europe. Des rois accusés de fomenter ou d'entretenir les haines intestines sur le territoire de la République dans l'espoir de l'affaiblir aux frontières, de détourner les citoyens d'un ordre politique constamment troublé et incapable d'assurer à chacun, contrairement à son projet avoué, la sûreté de sa personne et de ses biens, et de les jeter en définitive dans les bras de la royauté honnie. La guerre de Vendée et la piteuse expédition de Quiberon en sont les formes les plus virulentes. Mais les journaux thermidoriens n'ont de cesse de rappeler que ce danger est constamment accru par les menées subversives des " anarchistes », volontiers complices des agents de Pitt et des émigrés. Entendons sous ce terme d'abord les partisans de Robespierre, les jacobins, puis de plus en plus souvent à partir de germinal an III, les sans-culottes, les membres des comités de surveillance, des sociétés populaires, en somme les représentants des différentes instances du Gouvernement révolutionnaire de l'an II et du mouvement populaire. Cette configuration n'est du reste pas

$1795)$

(27) Boissy D’Anglas, Discours préliminaire au projet de constitution, BN Le38 1496 (23 juin (28) Ibid. 
propre à l'an III. On y retrouve le schéma de la triple conspiration dénoncée au printemps 1793 par les Girondins, avant leur éviction de la Convention ${ }^{29}$.

Haines politiques exprimées parfois dès le procès du roi en novembre et décembre 1792, mais aussi haines sociales exacerbées par les revendications populaires. Le discours thermidorien, lorsqu'il dénonce les « anarchistes », ne se fait pas faute de rappeler la haine vouée aux propriétaires pendant la Terreur, et de dénoncer la funeste et fausse opposition entretenue intentionnellement entre les riches et les pauvres. L'association de ces haines, qui sont significativement presque toujours exprimées au pluriel, à l'anarchie, tout au long des six derniers mois d'existence de la Convention thermidorienne, contribue à raviver perpétuellement le spectre de la guerre civile ${ }^{30}$.

L'anarchie ainsi dénoncée sans relâche cristallise en l'an III les peurs des thermidoriens pris entre le Charybde du royalisme et le Scylla des fantômes de la «tyrannie décemvirale ». Se construit alors une configuration qui tend à réunir sous la bannière de l'anarchie ces deux extrêmes dont prétendent également se prémunir les concepteurs de la nouvelle constitution. Prodrome d'une république de " l'extrême-centre " ${ }^{31}$ ? En tout cas mise en place d'un espace public ambivalent: ternaire et centré si l'on considère que les conventionnels occupent le centre d'un espace politique dont les extrêmes sont deux séditions également redoutables, celle du royalisme et celle des derniers sectateurs de Robespierre et de Marat; binaire et dichotomique dès lors qu'est affirmée sans ambiguïté l'identité de nature des extrêmes qui non seulement se rejoignent mais se confondent comme modalités d'un même danger, indifféremment désigné comme tyrannie, despotisme... ou anarchie ${ }^{32}$. C'est précisément à ce point que se comprend la formule adoptée en l'an $\mathrm{V}$ et qui fait écho à la manière dont Chénier exhortait ses collègues, le 6 messidor an III, à la Convention : « Soyez tous réunis pour la république, soyez tous réunis contre l'anarchie $»^{33}$.

(29) Marc Deleplace, L'Anarchie de Mably à Proudhon (1750-1850), op. cit., p. 132-145.

(30) Ibid., p. 150-158.

(31) Pierre Serna, La République des girouettes. 1789-1815 et au-delà. Une anomalie politique : la France de l'extrême centre, Paris, Champ Vallon, 2005.

(32) Marc Deleplace, op. cit., p. 132-145.

(33) M.-J. ChÉNIER, Discours à la Convention, 6 messidor an III (24 juin 1795), BN. 8-LE38-1507. Cette position de l'anarchie rejoint «l'ambiguité de l'unanimisme anti-terroriste de l'après thermidor », selon la formule de Bronislaw BACZKo, "L'Expérience thermidorienne », dans Colin Lucas (éd.) The French Revolution and the Creation of Modern Political Culture, Oxford-New York, 1989, tome 2, «The Political Culture of the French Revolution », p. 341 et suivantes. 
Mais Chénier nous rappelle aussi autre chose : la force de l'anathème jeté contre l'ennemi pour assurer la cohérence et la force du groupe qui le produit, notamment lorsque la cohésion socio-politique de ce groupe est rien moins qu'évidente. Rencontre de circonstance, comme d'autres groupes de cette sorte certes, le groupe des thermidoriens a pu trouver dans la désignation précise des « anarchistes »-précise non parce qu'elle aurait visé un groupe très nettement délimité, ce qui en aurait circonscrit fortement l'efficacité, mais au contraire parce que compréhensive de toute forme d'opposition à la Convention - le ciment dont il avait temporairement besoin ${ }^{34}$. Ciment qui survit à son efficacité immédiate, conjoncturelle, de la crise du printemps et de l'été de l'an III, alors que la Convention doit repousser successivement les assauts plus ou moins dangereux de la «queue » de Robespierre, en germinal et prairial, puis des royalistes en fructidor et vendémiaire an IV. Nous partageons ici le point de vue de Pierre Serna refusant que « la complexité à définir ce groupe, dont on ne peut cependant nier l'existence », le rende à l'inconsistance, mais qu'au contraire il « mérite que l'on mette à mal l'idée que l'on se fait de sa prétendue mollesse idéologique $»^{35}$. Nous pensons notamment que la question du serment civique de l'an V, comprise dans le prolongement de la politique thermidorienne, en rend compte à sa manière. Ainsi la proximité entre la mise en forme du serment et le coup d'État du 18 fructidor dont la dureté de la répression qui le suit « représente une claire illustration de cet extrême centre doublé d'un fort conservatisme républicain $»^{36}$, n'est-elle pas seulement contingente. Elle s'inscrit bien pour nous dans le mouvement de recherche «d'une façon d'édifier la stabilité souhaitée pour un régime républicain $»^{37}$. En revanche, de l'affirmation que « tout le programme et l'objectif de ce régime ont été [...] de fonder une politique du centre et de lui conférer un sens et une visibilité perceptibles, en un espace nouveau, ni à droite, ni à gauche », nous ne retiendrons sans restriction que l'expression qu'elle contient de l'originalité effective de ce régime, réservant notre jugement sur la nature de ce nouvel espace politique. Si nous retrouvons, aussi bien en l'an III qu'en l'an V, à travers notre exploration des notions « négatives » d'anarchie et

(34) Cette capacité de l'anarchie est donc liée non à la stabilité du désignant qui en eût au contraire invalidé l'effet, mais à sa plasticité à partir du moment où elle s'accompagne d'un processus de mise en cohérence conceptuel très abouti. Voir Marc Deleplace, op. cit.

(35) Pierre Serna, op. cit., p. 420.

(36) Ibid., p. 421.

(37) Ibid., p. 416. 
de haine, l'idée que « les extrêmes se touchent ou que les "royalistes" à bonnet rouge ou à cocarde blanche, se valent tous $»^{38}$, force nous est de constater que l'équivalence des extrêmes n'est jamais pleinement assurée, comme le montrent les ambiguïtés de la prestation du serment en l'an V, puis le débat de l'an VII.

Derrière l'évidente continuité entre discours thermidorien et politique directoriale, se profile en outre une épaisseur plus grande du substrat discursif et conceptuel, ainsi que des circonstances qui ont abouti à l'acceptation de la formule du serment. Nous distinguerons par commodité deux strates, dont les rapports avec l'émergence d'un serment de haine institué comme référence de formation de l'ordre politique en l'an V sont plus ou moins aisés à discerner dans l'archive, tant la circulation des mots est ici fluide. La première de ces strates, la plus apparente, est formée d'expériences concomitantes d'usages positifs de la haine dans le discours politique révolutionnaire. La seconde renvoie à des expériences antérieures qui posent le problème de la continuité entre philosophie politique, réflexion historique et morale du XVIII ${ }^{\mathrm{e}}$ siècle, et discours politique révolutionnaire.

La haine, élevée à la dignité paradoxale de point de ralliement pour les forces de progrès, comme l'on pourrait dire de manière anachronique, n'est pas un phénomène isolé dans la Révolution française. Tout d'abord, le serment civique de l'an V en lui-même : de tels serments, d'une portée plus ou moins générale, se renouvellent régulièrement depuis l'acte fondateur du jeu de paume le 20 juin 1789. Parmi ces serments, les serments de haine ne sont eux-mêmes pas ignorés. C'est ainsi que Mallarmé, envoyé en germinal an II comme représentant en mission dans la Meuse et la Moselle, dans des circonstances militaires et intérieures difficiles, fait placarder une proclamation « au nom de la République française une, indivisible et populaire » qui précise la place de chacun dans la France révolutionnaire et les actes par lesquels se concrétise l'adhésion à la Révolution en promettant «Égalité, fraternité aux sans culottes ; terreur aux aristocrates ; haine aux modérés $»{ }^{39}$ Et Thibaudeau évoque en l'an V, dans le débat sur l'extension du serment aux électeurs, un hypothétique

(38) Ibid., p. 419.

(39) Au nom de la République française, une, indivisible et populaire. Égalité, fraternité aux sans culottes ; terreur aux aristocrates; haine aux modérés. Mallarmé, présentant du peuple français, délégué par la Convention nationale dans les départements de la Meuse et de la Moselle, pour l'exécution des mesures de salut public et l'organisation du Gouvernement révolutionnaire [germinal an II ?]. BNF, Fol-LB41-4695. 
« serment prononcé dans l'Assemblée législative de haine à la République et aux deux chambres $»^{40}$.

En second lieu, la haine de la tyrannie, qui sous-tend le serment, s'affiche en novembre et décembre 1792 lors du procès du roi. Jean-Baptiste Mailhe, ouvrant la discussion, n'affirme-t-il pas : «Tous les Français partagent votre haine pour la tyrannie; tous abhorrent également la royauté $»^{41}$ ? Et Olivier Le Cour Grandmaison précise : « Ce discours est remarquable en ce qu'il use d'une rhétorique politique et affective qui place l'indignation et la haine au fondement de toute position légitime $»^{42}$. Mais ici, la haine positive puise également à d'autres sources, plus anciennes, plus discontinues sans doute dans leur rejeu au cours de la Révolution, mais non moins prégnantes cependant. La haine de la tyrannie relève, en effet, d'une expérience historique familière aux acteurs de la Révolution, au point que nous en retrouvons explicitement la trace dans leurs discours, celle de la République romaine et de sa haine de la royauté ${ }^{43}$. L'abbé René Aubert de Vertot, considéré par ses contemporains pour l'histoire de la république romaine qu'il publie en 1719 , mentionne à propos du renversement des Tarquins « cette haine générale que tous les Romains avaient contre le roi et même contre la royauté ». Il explicite cette attitude par la manière dont « un pouvoir despotique et cruel s'était élevé sur la ruine des lois et de la liberté », pouvoir despotique dont il mesure l'étendue à ce que « les différents ordres de l'État étaient également opprimés $»^{44}$. Ce passage de la haine d'un homme à la haine d'un principe est essentiel dans la valorisation positive de la haine dans l'espace public. La locution « haine publique » rend compte de ce glissement d'un ordre de chose à un autre. Elle peut en effet se comprendre d'abord négativement comme la haine du public envers une personne privée. Nicolas Coëffeteau montre ainsi, en 1646, comment « la haine du peuple romain s'accrut, et s'enflamma davantage contre Antoine $\aleph^{45}$, par suite des

(40) Corps législatif. Conseil des Cinq-Cents. Discours de Thibaudeau sur le serment proposé pour les électeurs, séance du 25 ventôse an V. BN 8-LE43-811, p. 7. Hypothétique dans la mesure où nous n'en avons encore retrouvé aucune trace.

(41) Cité dans Olivier Le Cour Grandmaison, op. cit., p. 240.

(42) Ibid., p. 241.

(43) Paul-Marius Martin, L'idée de royauté à Rome. 2, Haine de la royauté et séductions monarchiques : du IV siècle av. J.-C. au principat augustéen, Clermont-Ferrand, Adosa, 1994.

(44) René Aubert de Vertot, Histoire des révolutions arrivées dans le gouvernement de la république romaine, Livre I, Paris, F. Barois, 1719 p. 43-44.

(45) Nicolas COËFFETEAU, Histoire romaine, contenant tout ce qui s'est passé de plus mémorable depuis le commencement de l'empire d'Auguste, jusqu'à celui de Constantin le Grand, Seconde édition, Paris, H. Ruffin, 1646, p. 176, Livre I. 
manœuvres d'Octave. Cette « haine publique » peut ensuite décrire une haine sociale entre deux groupes, qui n'est plus le seul reflet des haines entre factions aristocratiques. Vertot relève ainsi « la haine du peuple pour tout ce qui portait le nom de patriciens $»^{46}$. Le discours thermidorien en l'an III dénoncera sans relâche de telles haines. Le renversement positif de cette haine se fait par exemple lorsque le ministre Quinette à la veille de l'anniversaire du 18 fructidor, en l'an VII écrit : « [Le matin du 18 fructidor, les Magistrats du peuple] voueront à la haine publique les réacteurs royaux et tous ceux qui, sous le masque hideux d'un faux patriotisme, tenteraient de renverser la constitution de l'an III $\gg{ }^{47}$. Et le passage des hommes au principe s'explicite à propos du serment de l'an V. « Non, ce n'est pas la haine des rois comme individus que la France désir dans le cœur de ses fonctionnaires : c'est la haine de la royauté en général comme fléau de la nature. C'est la haine surtout de la royauté en France où chacun de vous veut jouir de la liberté et de tous ses droits incompatibles avec les droits du trône $»^{48}$.

Reprenons maintenant le déroulement de la mise en place du serment. Dès la réunion des nouvelles assemblées directoriales, après que se fut séparée la Convention, la question d'un serment civique à exiger des représentants élus des administrations est agitée. Le principe en est adopté après discussion par le corps législatif le 22 frimaire an IV (13 décembre 1795), en vue de la cérémonie de commémoration de l'exécution du roi le $1^{\text {er }}$ pluviôse suivant. Ce principe est inscrit dans la loi le 23 nivôse an IV. La principale disposition en est la suivante : «Ce jour, à midi précis, le président de chaque conseil du Corps législatif prononcera un discours relatif à l'époque mémorable et recevra le serment des représentants du peuple, qui individuellement et à la tribune jureront haine à la royauté $\gg{ }^{49}$. Pour compléter cette mesure, le ministère de la Police de la République adresse aux administrateurs parisiens l'injonction suivante : «Citoyen, c'est demain, le $1^{\text {er }}$ pluviôse, $11 \mathrm{~h}$ du matin, que conformément à l'arrêté du Directoire exécutif du 25, en exécution de la loi du 23 de ce mois, vous

(46) René Aubert de Vertot, op. cit., p. 229.

(47) René Aubert de Vertot, op. cit., p. 229.

(48) Hymne à l'Être suprême. Discours sur le serment de haine à la royauté et à l'anarchie et imprécation contre les parjures. Pour la fête du 2 pluviôse VII de la République française, anniversaire du 21 janvier 1793 par le citoyen Mulot, ancien député de Paris à la première législature, professeur de belles lettres, membre du Lycée des Arts et de la société libre des sciences, lettres et arts de Paris [Mayence], BN 8-LB42-2058.

(49) Cité dans Corps Législatif. Conseil des Cinq-Cents. Motion d'ordre faite par Pierre Guyomar, séance du 17 nivôse an V. BN 8-Le43-669, p . 2. 
devez vous rendre à l'École militaire, et de là au champ de la Fédération, pour y exprimer par un serment solennel, la haine que vous avez vouée à la royauté, et l'attachement que vous avez manifesté pour la République, en acceptant les fonctions honorables que vous exercez $»{ }^{50}$. La formule n'en est donc pas encore formellement arrêtée. S'il comporte bien les deux volets que l'on retrouvera par la suite, liant haine de la royauté et attachement à la République, ils ne sont encore que juxtaposés et seule la royauté est vouée à l'exécration.

Or ce premier serment produit une réaction de refus, inattendue pour ses promoteurs si l'on en juge par le rapport fait à ce propos par Treilhard, futur directeur, au Conseil des Cinq-Cents le 18 ventôse an IV ( 8 mars 1796). Un corps de fonctionnaire a refusé, dans une proportion que nous ne connaissons pas mais suffisante pour susciter débat parlementaire, de prêter le serment: ce sont les juges ${ }^{51}$. Cette éventualité n'avait de toute évidence pas été anticipée par les législateurs, et Treilhard s'en explique. «Si l'on nous demande pourquoi le Corps Législatif n'a pas prononcé de peine contre le fonctionnaire républicain qui balancerait à prêter serment de haine à la royauté, nous répondrons aussi : nous n'avons pas pu croire qu'un pareil fonctionnaire dût exister $\gg^{52}$. Passée la surprise, le législateur réagit en des termes qui avancent les premiers éléments d'une acclimatation de la haine comme principe républicain. Il commence par un retour sur les raisons de cet acte inouï, exposant la réalité d'un espace public d'affrontement dans lequel les haines se répondent les unes aux autres. «Les hommes mêmes que vous avez choisis pour être les juges de la nation refusent de maudire la royauté, et ce sentiment de haine que le peuple français voue aux tyrans, ils le vouent à la république ». Puis, réclamant que le sol de la République soit purgé de « ces vils fauteurs de nos plus cruels ennemis », il trace les principales lignes de la conduite à tenir : «Quant à nous, marquons chaque pas du

(50) Paris, le 30 nivôse an 4 de la République Française une et indivisible. Le ministre de la police générale de la République à... [signé S et F Merlin (de Douai)] BN, 4-LE SENNE (13).

(51) Le débat qui s'ouvre en nivôse an VI sur la révision des jugements rendus en haine de la République, dans la période qui précéda le 18 fructidor an V, peut également attester de l'ampleur effective de ce refus. Voir : Corps législatif. Conseil des Cinq-Cents. Rapport fait par Cholet au nom d'une commission spéciale sur un message du Directoire exécutif concernant la révision des jugements rendus en haine de la République pendant la réaction royaliste, 29 nivôse an VI, réimprimé pour être distribué à nouveau conformément à l'arrêté du Conseil des Cinq-Cents du 21 thermidor an VII.

(52) Conseil des Cinq-Cents. Rapport fait au nom de la commission nommée pour rendre compte du message du Directoire exécutif relatif aux juges qui ont refusé de prêter serment de haine à la royauté. Par Treilhard, séance du 18 ventôse an IV, BN 8-LE43-145. 
Corps Législatif d'une fermeté réfléchie et d'une sagesse que rien ne saurait ébranler ; travaillons constamment et d'une main assurée à compléter l'édifice de notre liberté et ne laissons pas à nos ennemis même l'espoir de nous voir faire le bien d'une manière injuste et arbitraire ${ }^{53} »$. Il prend ainsi bien garde de sembler céder au vertige de l'arbitraire despotique ni réclamer les échafauds de la Terreur. Il lie aussi, pour la première fois, de manière explicite, l'achèvement de «l'édifice de la liberté » à la haine du despotisme, substituant l'affrontement des principes à celui des personnes, adossant la liberté à la haine et enfermant celle-ci dans la loi de sorte qu'elle n'engendre point la vengeance mais garantisse au contraire l'ordre public, de tel sorte que soit atteint ce but paradoxal : faire le bien d'une manière juste par un serment de haine. Le Conseil des Cinq-Cents adopte alors la proposition suivante :

«Art. I : Aucun membre des autorités constituées de la République ne pourra désormais entrer en exercice de ses fonctions, sans avoir préalablement prêté serment de haine à la royauté : ceux qui exerceraient leur fonction sans avoir prêté ce serment seront punis de la peine de déportation.

Art. II : Ceux des juges qui n'ont pas encore prêté le serment de haine à la royauté, le prêteront dans les trois jours de la publication de la présente loi ».

Non seulement la royauté est toujours seule en cause dans le camp de la tyrannie qu'il s'agit de combattre, mais encore l'attachement à la République s'efface devant la haine vouée à cette tyrannie. Ainsi une loi de la République subordonne-t-elle l'exercice des fonctions publiques à un principe de haine.

Mais ce serment ne suffit pas à prémunir la République contre tous ses ennemis. Depuis l'an III, il est clair que l'anarchie, qui est le despotisme de tous, peut surpasser en virulence le despotisme d'un seul. Une dernière étape est donc franchie à l'approche de l'anniversaire de l'exécution du roi en l'an V lorsque Philippes-Deleville demande que la haine à l'anarchie soit adjointe à la haine à la royauté. Il amplifie ainsi la demande formulée par Guyomar quelques jours auparavant. Ce dernier avait en effet souhaité que soit pérennisé le serment prononcé lors de la cérémonie du $1^{\text {er }}$ pluviôse an IV, au motif que le renouvellement annuel d'une partie du Corps législatif justifiait que l'on exigeât des nouveaux venus les mêmes démonstrations d'attachement à la République que celles des 
premiers législateurs de la toute nouvelle République. Comme Treilhard, il n'envisage encore que le serment de haine à la royauté, et plus que lui, il explicite ce qui fait d'un serment de haine à la royauté un serment évident d'attachement à la République. Il justifie dans le même mouvement que le serment exigé soit bien en priorité celui de haine à la royauté. « Jurer tous les ans haine à la royauté, c'est prêter tous les ans serment de fidélité à la République, c'est jurer de maintenir la constitution républicaine élevée sur les débris du trône, c'est rassurer le républicain inquiet sur notre fidélité à conserver intact le dépôt qui nous est confié par le pacte social $\gg{ }^{54}$.

Philippes-Deleville lui emboîte le pas tout en renouant avec le discours thermidorien sur l'anarchie. Dans son argumentaire, il explicite le sens de sa démarche par le besoin de mettre en cohérence plusieurs décisions successives qui concourent toutes au même but : établir et garantir la constitution républicaine ${ }^{55}$. Il insiste sur l'utilité de faire coïncider de manière organique ce qui n'était jusque-là que juxtaposé : la haine à la royauté et la fidélité à la République, entendons non à la République comme principe mais à la République comme régime, à la constitution de l'an III. Ce faisant, il établit les prémisses d'un débat qui atteindra son acmé en thermidor de l'an VII et qui touche à la nature même de la République instituée par la constitution de l'an III, bien que son but avoué soit au contraire d'éteindre toute contestation de ce régime. Et cela précisément parce qu'il laisse pendante la question de l'adéquation effective entre ce régime et le principe républicain. Pour justifier sa démarche, il retrouve les accents de thermidor, lorsqu'il fallait à la fois se garder des suppôts du despotisme royal et de ceux de la tyrannie décemvirale. « Il faut que ce serment, qui ferme la porte de Rome aux Tarquin ne la rouvre pas aux Catilina, et pour parler sans figure, il nous faut un serment qui, en excluant pour toujours la royauté de notre territoire, en expulse à jamais les tyrans de toutes les couleurs $»^{56}$. Il amorce une distinction nécessaire

(54) Corps Législatif. Conseil des Cinq-Cents. Motion d'ordre faite par Pierre Guyomar, séance du 17 nivôse an $V$. BN 8-Le43-669, p. 3.

(55) «Une résolution du conseil, convertie en loi par le Conseil des Anciens, vient de faire coïncider le jour du serment de haine à la royauté avec le calendrier républicain ; je viens vous proposer aujourd'hui de coordonner ce serment avec la République et la constitution. » Corps législatif. Conseil des Cinq-Cents. Motion d'ordre de Philippes-Deleville, membre du conseil des Cinq-Cents et député du Calvados, séance du 22 nivôse an V. BN 8-LE43-678, p. 2.

(56) Ibid., p. 2. La référence à l'histoire romaine, figure de rhétorique tellement évidente que l'auteur la signale lui-même pour l'abandonner aussitôt, matérialise également pour nous le fil de ces continuités que nous avons voulu esquisser entre discours historique, philosophique et moral du $\mathrm{XVII}^{\mathrm{e}}$ et du XVIII ${ }^{\mathrm{e}}$ siècle et possibilité d'une haine positive dans le discours républicain de la Révolution. 
pour légitimer le régime républicain en le séparant de l'anarchie ${ }^{57}$. Il établit nettement le départ entre deux formes de haines, qui, pour sembler avoir le même objet, n'en sont pas moins opposées de nature. «Vous le sentez tous, citoyens collègues, en jurant simplement haine à la royauté, nous ne jurons rien que Marat, Robespierre, et leurs successeurs et leurs sicaires anarchistes n'aient juré et ne jurent encore volontiers ». Aussi « ce n'est pas assez d'avoir aboli le gouvernement royal et de le haïr, comme je l'ai dit, à la manière des brigands et des anarchistes ; il faut joindre à notre haine sainement entendue un attachement spécial et durable au gouvernement républicain et à la charte constitutionnelle qui nous le garantit ${ }^{58} »$. La haine est « saine » non seulement parce qu'elle est le levier de la liberté, mais en ce qu'elle contribue à assainir le corps politique républicain en repoussant ceux qui, par attachement à la royauté, se refusent à s'y associer, et surtout ceux qui tenteraient, sous couvert de cette même haine de la royauté, de le gangrener de l'intérieur.

La proposition de Philippes-Deleville est adoptée le 24 nivôse an V (13 janvier 1797$)^{59}$.

\section{Prestation, débats et abandon (an V-an VII)}

Les prestations successives du nouveau serment permettent d'expliciter à quel niveau se noue la question de la haine comme fondation de l'ordre politique, dans la logique du propos du citoyen Barry, en faisant retour sur le couple essentiel amour/haine. La haine ne s'oppose pas à l'amour mais en forme le contrepoint nécessaire à l'établissement de la véritable liberté, à la garantie du bonheur, qui est le but ultime de la formation des sociétés politiques. «Le but de cette institution sacrée [le serment civique] est de propager et de consolider l'horreur de la tyrannie et de la transmettre d'âge en âge [...]. Peut-on en effet aimer la liberté sans haïr la royauté son ennemie implacable $»^{60}$ ? Il est alors possible d'associer sans restriction la haine à la réalisation et la conservation du pacte social, dans des formulations qui n'ignorent pas le souvenir de la référence au droit naturel, alors même que cette référence a été volontai-

(57) Marc Deleplace, op. cit.

(58) Op. cit. BN 8-LE43-678, p. 3.

(59) Initialement prévu pour les administrations, il est étendu aux électeurs en ventôse an V, puis aux prêtres le 19 fructidor an V.

(60) Discours prononcé à la fête du 2 pluviôse an $5^{\mathrm{e}}$ de la république [...] Par le citoyen Barry, président de l'administration municipale du canton de Toulon, BN 8-LB42-1233. 
rement exclue de l'acte constitutionnel ${ }^{61}$. « Que cet acte solennel et unanime redouble notre énergie, notre amour pour la patrie, pour la liberté, pour la constitution de l'an III, notre port de salut, et qu'il resserre entre nous les doux liens de l'Égalité et de la Fraternité $\gg{ }^{62}$. Le serment retrouve ainsi un dernier antécédent, dont la proximité semblera la moins évidente avec notre sujet, mais non moins décisive : la haine de Dieu pour le péché, abondamment développée par la littérature ecclésiastique des XVII ${ }^{\mathrm{e}}$ et XVIII ${ }^{e}$ siècles. L'oratorien Senault, dans son traité des passions publié en 1641 et souvent réimprimé par la suite, concède dans un premier temps que l'amour et la haine s'opposent « car l'amour est obligeant, et la haine est malfaisante, l'amour est généreux et prend plaisir à pardonner, la haine est lâche et ne médite que des vengeances, l'amour donne la vie à ses ennemis, la haine procure la mort à ses plus fidèles amis, et il semble qu'on accorderait plutôt le vice avec la vertu, que l'amour avec la haine». Mais c'est pour ajouter immédiatement, comment ces deux passions peuvent, selon l'objet auquel elles s'appliquent, se compléter au lieu de s'opposer : « L'amour du bien est une haine du mal, et cette même passion qui a de la douceur pour ceux qui l'obligent, a de la sévérité pour ceux qui l'offensent $»^{63}$. C'est cette continuité de l'amour à la haine qui organise en dernière instance le serment de l'an $\mathrm{V}$ et rend la haine acceptable dans l'espace public, et plus encore fondatrice de cet espace.

Le lien entre la prestation du serment de haine et l'affirmation de la souveraineté libre du peuple français ne cesse dès lors d'être réaffirmé. Ainsi, en l'an VI, le président du Conseil des Anciens peut-il s'écrier : «Peuple français! Reçois le serment que nous allons prononcer. En jurant la haine à la royauté, haine à l'anarchie, attachement et fidélité à la constitution de l'an III, nous jurons de consolider le pacte solennel que tu as placé comme un mur d'airain entre toi et tous les genres d'oppression; nous jurons d'affermir et de défendre, même au péril de nos jours la liberté et les droits que tu as reconquis $»^{64}$. Et son homologue du Conseil des Cinq-Cents, en réactivant l'image négative de la haine

(61) Voir note 8 .

(62) Citoyen BARRY, op. cit., BN 8-LB42-1233.

(63) Jean-François Senault, op. cit., p. 58-59. Nous retrouvons ainsi, du religieux au politique, une problématique développée en d'autres termes par Dale VAN KLEY : Les origines religieuses de la Révolution française 1560-1791, Paris, Éditions du Seuil, "L'Univers historique », 2002. La démonstration de la circulation des argumentaires retient particulièrement notre attention.

(64) Conseil des Anciens. Discours prononcé par Rousseau, président du Conseil des Anciens, pour l'anniversaire du 21 janvier et la prestation du serment de haine à la royauté et à l'anarchie, 2 pluviôse an 6, BN 8-LE45-758. 
comme discorde civile nous permet de saisir la dialectique qui attribue à la haine dépassionnée du serment la capacité à éteindre les haines furieuses des ennemis de la République, des sectateurs du fanatisme et de l'anarchie.

«Pour nous, toujours fermes dans la voie du républicanisme et de nos devoirs, nous allons prêter ce serment que les ennemis de la patrie savent bien n'être pas vain. Ce serment consacrera cette nouvelle enceinte, il lui imprimera le caractère qui dans les grandes catastrophes de la révolution détermina constamment le succès pour la liberté [...]. Nous la consacrons à la souveraineté du peuple sous les auspices de la paix continentale. Puisse cette heureuse circonstance être le présage du calme et de la sagesse de nos délibérations, de notre union, dont l'exemple efface les souvenirs douloureux, adoucisse les haines, arrête les vengeances ! que ces guirlandes et ces fleurs soient le symbole de la prospérité du peuple, de l'aisance et du bonheur dans les familles ! Mais tant de biens ne peuvent naître que de l'amour de la patrie et de la fidélité à remplir le serment que je vais prononcer $»^{65}$.

Le serment de haine devient alors la clé de voûte de l'édifice social en garantissant la collaboration harmonieuse des différents organes de l'État. « Voyez quels efforts les membres du Directoire exécutif font pour le maintien et la gloire de la République, voyez l'harmonie qui règne au sein des deux conseils dans les plans conservateurs de la République Voyez l'accord des administrateurs, des juges et de toutes les classes de fonctionnaires pour arriver au même but $»^{66}$. Et le citoyen Mulot de se demander, relativement à la mission des «fonctionnaires des départements nouvellement formés sur les rives du Rhin »: "pouvons-nous remplir nos devoirs si le sentiment généreux de la haine de la royauté n'est pas dans nos cœurs $\|{ }^{67}$ ? Nous ne sommes pas loin de retrouver ici, dans un contexte par ailleurs bien différent, l'approche que propose Sophie Wahnich de la question de la Terreur, lorsqu'elle explique « qu'il s'agit pour les révolutionnaires à la fois d'entendre les risques de violence et de dislocation de la société du fait de la circulation rapide des émotions

(65) Conseil des Cinq-Cents. Discours prononcé par J. Ch. Bailleul, président du Conseil des Cinq-Cents, le 2 pluviôse répondant au 21 janvier (ancien style), avant la prestation du serment de haine à la royauté et à l'anarchie, séance du 2 pluviôse an VI. BN 8-LE43-1713.

(66) Hymne à l'Être suprême. Discours sur le serment de haine à la royauté et à l'anarchie et imprécation contre les parjures. [...] par le citoyen Mulot [...] [Mayence], BN 8-LB42-2058.

(67) Ibid. Souligné par nous. 
et de les contrôler justement par l'activité symbolique dont participent les discours, en particulier le discours de la loi $\|^{68}$. Mais la question est ici de dénier une violence que le moment terroriste inciterait plutôt à poser comme fondatrice ${ }^{69}$.

La formule du serment embarrasse cependant, mais pas en raison de la haine qu'elle prescrit. C'est l'objet de cette haine étendu à l'anarchie qui trouble le républicain. Dans le contexte tendu qui précède les élections de l'an V, devant le danger royaliste toujours plus pressant, le serment conserve de ce fait une dissymétrie que son auteur n'avait pas voulue. En témoigne le discours prononcé par le Président du Conseil des Cinq-Cents, le 2 pluviôse (21 janvier 1797). Il exhorte d'abord les représentants du peuple conformément à l'esprit du serment : «Allumez et nourrissez de toute part le feu sacré de la liberté et proclamez sans cesse la haine de la royauté et l'horreur de l'anarchie $»^{70}$. Mais il réserve en fait à la royauté seule la haine qu'il s'apprête à jurer. Et lorsque vient le moment de l'explication, le déséquilibre s'aggrave. C'est à la royauté seule que se trouve opposée la République. « Haïr la royauté c'est aimer le peuple, c'est sentir la dignité de l'homme, c'est rendre hommage à la véritable souveraineté, c'est vouloir la république ». Et c'est cette même royauté dont la haine qu'elle voue impitoyablement à la République justifie en retour celle que l'homme libre est en droit de lui porter. " C'est pour elle [royauté], représentants du peuple, que l'on déverse sur vous à flots intarissables la haine et le mépris ». L'introduction de l'anarchie ne se fait que tardivement, et sur le mode de la déploration, redonnant à la haine toute sa charge négative. «En vain la raison dit à l'homme : Rien de trop, les passions l'entraînent d'un excès à un excès opposé : et dans un pays surtout, arène ensanglantée de haines et de réactions, où les institutions publiques ne sont pas encore fortement enracinées, tout marche ou rétrograde rapidement. Ce qui n'était qu'une opinion se change en un sentiment profond et actif : et les sentiments se dénaturent,

(68) Sophie Wahnich, op. cit. L'analogie que nous évoquons ne signifie pas que nous occultons la rupture de thermidor sur laquelle nous nous sommes exprimé plus haut, mais que la question de l'articulation de ces deux moments de la Révolution, Terreur et Directoire, nous paraît mériter réexamen.

(69) «La nature profonde de la terreur, c'est donc bien la violence punitive, réparatrice et préventive de la politique du peuple élevée, du fait de la guerre et du poids spécifique de la sansculotterie dans Paris, à l'éminente dignité d'un principe nouveau imposé aux élites politiques ». Roger Dupuy, op. cit., p. 137.

(70) Corps législatif. Conseil des Cinq-Cents. Discours prononcé pour l'anniversaire du 21 janvier et la prestation du serment de haine à la royauté et à l'anarchie, prononcé par Riou (du Finistère), Président du Conseil des Cinq-Cents, 2 pluviôse V, BN 8-LE43-696. 
affaiblis ou exagérés, par l'esprit de parti : la douceur devient faiblesse, le courage férocité : on hait par commisération, on assassine par humanité, on venge des victimes par des victimes [...]». Il se ressaisit cependant pour retrouver le sens de la démarche amorcée un an auparavant. «Mais que les souvenirs des crimes révolutionnaires n'efface pas celui des crimes de la royauté : que votre haine contre les terroristes ne s'étende pas aux républicains : jurez comme nous haine à la royauté, haine à l'anarchie ; marchez d'un pas ferme entre ces deux extrémités également funestes. C'est dans un juste milieu que vous trouverez la liberté et le bonheur $[\ldots] »^{71}$.

La position de la haine dans le discours républicain directorial, hormis sa répétition stéréotypée dans la formule du serment, n'en apparaît pas moins bien fragile et difficile à tenir. La volonté d'étendre le serment à «toutes les formes de tyrannies » en y incluant la haine à l'anarchie, dont on hésite pour savoir si elle ne risque pas de recouvrir la haine de la République elle-même au prétexte d'en rejeter les excès passés, si elle réalise le souhait des thermidoriens de sortir de la Terreur ${ }^{72}$ et fonder un gouvernement représentatif, en est la cause évidente. Avant même que ne soit ouvertement attaqué cette formulation par Jourdan en thermidor de l'an VII, nombreux sont les discours qui en gauchissent le sens en conservant à la royauté sa valeur d'objet premier de haine. Rejoue ici un autre argumentaire élaboré en l'an III qui, au lieu d'établir une analogie de nature entre la royauté et l'anarchie, faisait de cette dernière l'instrument de la première. Une position reprise par le citoyen Mulot lors de la dernière prestation du serment, en pluviôse an VII. Il met longuement l'accent sur la haine à la royauté, affirmant qu'elle n'est « autre chose cette haine que le juste sentiment que l'homme doit avoir de sa propre dignité, En effet, qui de nous en descendant au fond de son âme, n'y trouve pas écrite, en caractères indélébiles, la condamnation de la royauté, de ce régime où l'on n'a que des maîtres et point de patrie $»^{73}$ ? Il poursuit en rappelant ce qu'est ce « système essentiellement oppressif, contraire aux vœux de la nature, contraire aux droits éternels de l'homme, et dont tout le prestige s'évapore au creuset de l'expérience ». L'anarchie n'intervient alors que comme l'instrument possible de ce système honni, et Mulot dénonce les hommes qui forment « le projet horrible et constant de nous ramener à la royauté par l'anarchie ». Et de conclure : « Nous qui aimons la France, sa

(71) Ibid.

(72) Bronislaw Baczko, Comment sortir de la terreur, Paris, Gallimard, 1989.

(73) Citoyen Mulot, op. cit. 8-LB42-2058. 
gloire et notre bonheur, nous jurerons spontanément haine à la royauté et à ses instruments les plus funestes, le fanatisme et l'anarchie $[\ldots] \gg^{74}$.

C'est à partir de cette disparité entre les deux termes honnis que Jourdan puis Montpellier ouvrent le débat qui, du 6 au 8 thermidor an VII (25-27 juillet 1799), au plus fort de la poussée «néojacobine », aboutit finalement à l'abandon du serment de haine à l'anarchie. Un serment dont les orateurs montrent successivement qu'il est inepte, si l'on admet que l'anarchie est l'absence de tout gouvernement (car qui pourrait jurer haine à quelque chose qui n'existe pas ?), et qu'il n'est en fait qu'une concession faite aux royalistes dans la triste période qui précéda les élections de l'an V. Concession dangereuse, dès lors que Jourdan peut démontrer que derrière l'anarchie se profile en réalité la République elle-même, et non pas la république sanglante de la tyrannie jacobine mais bien la république directoriale. D'où il conclut qu'être républicain c'est être anarchiste et qu'il faut donc supprimer cette partie du serment ${ }^{75}$. De fait c'est tout le serment qui est réécrit, et significativement pour notre propos, ce n'est pas seulement l'anarchie qui disparaît du nouveau texte mais bien la haine elle-même. « Je jure fidélité à la république et à la constitution de l'an III : je jure de m'opposer de tout mon pouvoir au rétablissement de la royauté en France et à celui de toute espèce de tyrannie ${ }^{76}$. Tel se présente le serment civique, adopté le 23 thermidor (10 août 1799), pour être prononcé, cette fois, à l'occasion de la fête de la fondation de la République, sur l'autel de la patrie.

Faut-il s'étonner dans ces conditions de l'absence de postérité de cette tentative pour fonder l'ordre politique républicain sur un serment de haine, fut-elle haine de la tyrannie ? En effet, nous avons cherché en vain des traces de cette expérience dans le cheminement républicain du XIX siècle, alors même que, selon Claude Nicolet, la III ${ }^{\mathrm{e}}$ République puise largement dans 1'héritage des dernières années de la Révolution ${ }^{77}$. C'est bien davantage le souvenir des haines révolutionnaires ou contre-révolutionnaires, réactivé régulièrement par l'actualité politique, entretenu par l'historiographie et la littérature, qui demeure. S'il reste bien des traces

(74) Ibid.

(75) Marc Deleplace, op. cit., BN p. 121-129.

(76) Fête de la fondation de la République. Programme [signé Quinette] Fructidor an VII, BN, 4-LE SENNE (28).

(77) Claude Nicolet, L'idée républicaine en France 1789-1924, Paris, Gallimard, 1982. Pierre Serna écrit pour sa part : «Tout le XIX ${ }^{\mathrm{e}}$ siècle se trouve dans la matrice directoriale », op. cit., p. 415 . 
du serment, c'est précisément cette partie du serment que Jourdan et ses amis s'étaient évertués avec succès à déconsidérer en thermidor de l'an VII. L'Événement, fondé sous l'impulsion de Victor Hugo le $1^{\mathrm{er}}$ août 1848, dans l'ombre portée des journées de juin, ne formulait-il pas comme épigraphe : «Haine vigoureuse de l'anarchie, tendre et profond amour du peuple »?

Marc Deleplace

EA 2616-CERHIC (Centre d'étude et de recherche en histoire culturelle) Université de Reims-Champagne-Ardenne

IUFM Champagne-Ardenne

23 rue Clément Ader

51100 Reims

deleplace.marc@free.fr 